\title{
Storing keV Negative Ions for an Hour: The Lifetime of the Metastable ${ }^{2} P_{1 / 2}^{o}$ level in ${ }^{32} \mathrm{~S}^{-}$
}

\author{
E. Bäckström, ${ }^{1, *}$ D. Hanstorp, ${ }^{2}$ O. M. Hole, ${ }^{1}$ M. Kaminska, ${ }^{1,3}$ R. F. Nascimento, ${ }^{1}$ M. Blom, ${ }^{1}$ M. Björkhage, ${ }^{1}$ A. Källberg, ${ }^{1}$ \\ P. Löfgren, ${ }^{1}$ P. Reinhed, ${ }^{1}$ S. Rosén, ${ }^{1}$ A. Simonsson, ${ }^{1}$ R. D. Thomas, ${ }^{1}$ S. Mannervik, ${ }^{1}$ H. T. Schmidt,${ }^{1, \dagger}$ and H. Cederquist ${ }^{1}$ \\ ${ }^{1}$ Department of Physics, Stockholm University, AlbaNova, SE-106 91 Stockholm, Sweden \\ ${ }^{2}$ Department of Physics, University of Gothenburg, SE-412 96 Göteborg, Sweden \\ ${ }^{3}$ Institute of Physics, Jan Kochanowski University, 25-369 Kielce, Poland
}

(Received 16 December 2014; revised manuscript received 6 February 2015; published 6 April 2015)

\begin{abstract}
We use a novel electrostatic ion storage ring to measure the radiative lifetime of the upper level in the $3 p^{5} P_{1 / 2}^{o} \rightarrow 3 p^{52} P_{3 / 2}^{o}$ spontaneous radiative decay in ${ }^{32} \mathrm{~S}^{-}$to be $503 \pm 54 \mathrm{sec}$. This is by orders of magnitude the longest lifetime ever measured in a negatively charged ion. Cryogenic cooling of the storage ring gives a residual-gas pressure of a few times $10^{-14} \mathrm{mbar}$ at $13 \mathrm{~K}$ and storage of $10 \mathrm{keV}$ sulfur anions for more than an hour. Our experimental results differ by $1.3 \sigma$ from the only available theoretical prediction [P. Andersson et al., Phys. Rev. A 73, 032705 (2006)].
\end{abstract}

We have constructed an electrostatic ion storage ring for operation at cryogenic temperatures [1]. This allows storage of $\mathrm{keV}$ ions for extended periods of time [2], and, as will be demonstrated here, measurements of lifetimes of metastable states in negative ions in a completely new time domain ranging up to several minutes and beyond.

Negative atomic ions are unique quantum systems. Because of the absence of a long-range $1 / r$ potential, there are only one or a few bound quantum states with typical binding energies below a few eV. Direct interactions between the electrons-so-called electron correlation effects-thus, play much more important roles than in positively charged or neutral systems. This sensitivity makes measurements of long lifetimes in negative ions important benchmarkers for many-body treatments of electronic interactions in atomic systems [3-5].

The ${ }^{2} P_{3 / 2}^{o}$ ground-state level in ${ }^{32} \mathrm{~S}^{-}$is bound by 2.077 104 1(7) eV with respect to the neutral atom [6]; see Fig. 1. The fine structure splitting of the ${ }^{2} P_{J}^{o}$ term is 0.0599507 (4) eV [7]. The ${ }^{2} P_{1 / 2}^{o}$ upper level is metastable as electricdipole transitions to ${ }^{2} P_{3 / 2}^{o}$ are forbidden by quantummechanical selection rules. Instead, the decay is dominated by magnetic dipole (M1) transitions [8]. The only known examples with allowed electric-dipole transitions between truly bound states, i.e., states bound with respect to the ground state of the atom, in negative ions are $\mathrm{Os}^{-}$[9], $\mathrm{Ce}^{-}$ [10], and $\mathrm{La}^{-}$[11]. Recently, $\mathrm{La}^{-}$has attracted interest as a strong candidate for laser cooling of negative ions using the allowed ${ }^{3} F_{2}^{e} \rightarrow{ }^{3} D_{1}^{o}$ transition $[11,12]$.

Published by the American Physical Society under the terms of the Creative Commons Attribution 3.0 License. Further distribution of this work must maintain attribution to the author(s) and the published article's title, journal citation, and DOI.
Lifetimes of extremely long-lived metastable states may, under certain conditions, be determined even if the observation time is strongly limited in relation to the lifetime to be measured. By storing ions in magneto-optical traps for seconds only, it was possible to accumulate sufficient amounts of neutral metastable atoms and to implement efficient fluorescence-based detection schemes to measure the ${ }^{3} P_{2}$ lifetimes in ${ }^{88} \mathrm{Sr}$ and ${ }^{24} \mathrm{Mg}$ to be $520_{-140}^{+310} \mathrm{sec}$ [13] and $2050_{-110}^{+140} \mathrm{sec}$ [14], respectively. A magneto-optical trap was also used when the ${ }^{3} \mathrm{~S}_{1}$ lifetime in ${ }^{4} \mathrm{He}$ was measured to be $7870 \pm 510 \mathrm{sec}$ [15]. This is, to our knowledge, the longest lifetime ever measured for a neutral atomic system. Nuclear lifetimes of thousands and millions of years and beyond have been measured using macroscopic amounts of radioactive material.

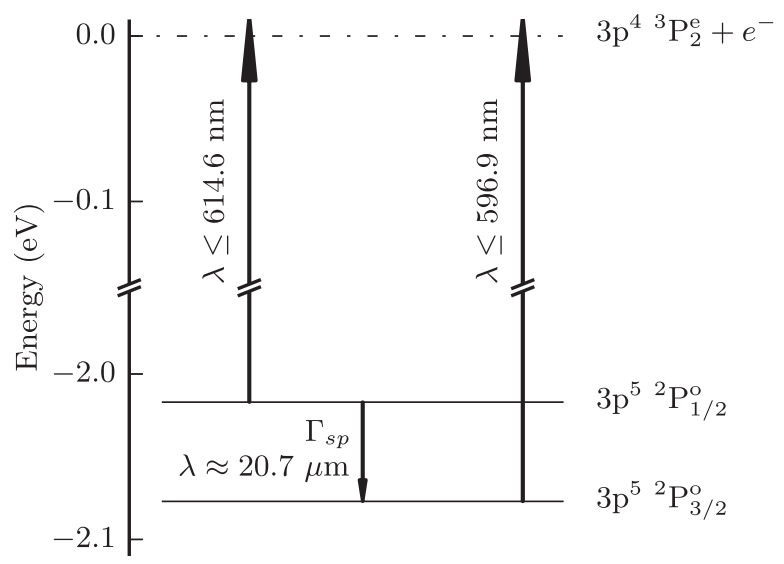

FIG. 1. The binding energies of the only bound quantum states in the ${ }^{32} \mathrm{~S}^{-}$ion. Ions in the upper fine structure level can only decay by forbidden radiative transitions with the rate $\Gamma_{\mathrm{sp}}$ expected to be dominated by magnetic dipole $(M 1)$ transitions at $20.7 \mu \mathrm{m}$. The longest wavelengths that may result in photodetachment for the two levels are indicated. 
Because of their charge and large polarizabilities, negative ions cannot, like neutral atoms or radioactive species, be concentrated in small volumes without affecting the very decay behavior under investigation. Further, they typically emit in the infrared, and with one exception [11,12], there are no strong candidates for laser cooling of negative ions. This means that photon counting will be very inefficient and that fluorescence-based methods cannot be used. The decay of negative ions may, instead, be efficiently probed by means of time-resolved photodetachment of individual ions in small and dilute samples that move with high velocity in a laboratory frame of reference. As the neutral photodetachment products then will have essentially the same velocity as the ions, they may be counted with high efficiency. This requires an ion-beam trap $[16,17]$ or an ion storage ring [18-20] and storage over extended times such that instrumental limitations can be separated from the intrinsic lifetime to be measured.

Lifetimes of metastable states in negative ions have been measured previously. One example is the lifetime of the autodetaching $1 s 2 s 2 p^{4} P_{5 / 2}^{o}$ level in ${ }^{4} \mathrm{He}^{-}$, which has been measured in magnetic [21] and electrostatic [22] ion storage rings and in electrostatic ion-beam traps $[23,24]$. As shown by these experiments, electrostatic devices do not mix fine structure levels in any significant way for $\mathrm{He}^{-}$ $[21,22]$. Further, cryogenic cooling was shown to eliminate all measurable effects of the black-body radiation field for the ${ }^{4} P_{5 / 2}^{o}$ levels in ${ }^{4} \mathrm{He}^{-}$with its inherent lifetime of a few tenths of a millisecond [24]. The only previous lifetime measurements of a truly bound metastable state are for the ${ }^{2} P_{1 / 2}^{o}$ level in $\mathrm{Te}^{-}[8,25]$ and in $\mathrm{Se}^{-}$[8]. These were measured in a room-temperature magnetic storage ring (pressure $10^{-11}-10^{-12}$ mbar) to have lifetimes of up to a few seconds.

Our newly developed ion storage ring is cooled to $13 \mathrm{~K}$, which gives an extremely low pressure of a few times $10^{-14}$ mbar and an estimated residual-gas density of about $2 \times 10^{4} \mathrm{~cm}^{-3}[2]$. This allows storage of negative ions for an hour and longer. In the following paragraphs, we will briefly describe the new storage ring setup. We will explain how lifetime measurements are performed by means of laser probing of the metastable population in circulating $10 \mathrm{keV}^{32} \mathrm{~S}^{-}$ion beams and how we take beam loss due to electron detachment in the small amounts of residual gas into account. In addition, we present measurements on $\mathrm{Te}^{-}$ and $\mathrm{Se}^{-}$and demonstrate that our new method is a factor of 5 more precise than the previous ones $[8,25]$ due to our much longer storage times.

The rate of the spontaneous

$$
\mathrm{S}^{-}\left(3 p^{52} P_{1 / 2}^{o}\right) \rightarrow \mathrm{S}^{-}\left(3 p^{52} P_{3 / 2}^{o}\right)+h \nu
$$

radiative decay was measured by means of photodetachment using two different photon energies. The first was chosen such that only ions in the excited $3 p^{52} P_{1 / 2}^{o}$ level were photodetached, while the second was chosen to photodetach ions in both levels; see Fig. 1. This is repeated at different times after injection and on the same ensemble of stored ions for a given laser-photon energy. This allows us to follow beam losses and the population of the metastable level as functions of time. The procedure is repeated for many, typically ten, consecutive injections.

Negatively charged ${ }^{32} \mathrm{~S}$ sulfur ions (nuclear spin zero) were produced in a cesium sputter ion source (SNICS II [26]) which was mounted on the ion injection platform for one of the ion storage rings at the DESIREE facility [1,2]. A FeS cathode was used in the sputter source, and the ions were accelerated to $10 \mathrm{keV}$ before entering a bending magnet with resolving power $10^{3}$ to select the ${ }^{32} \mathrm{~S}^{-}$beam. The ion beam was then chopped into $20 \mu$ s long pulses and guided over a distance of about $6 \mathrm{~m}$ before injection in the ion storage ring with a circumference of $8.6 \mathrm{~m}$. The revolution time in the ring was $\approx 35 \mu \mathrm{s}$, and after a few thousand turns, the $\mathrm{S}^{-}$ions were distributed over the whole circumference of the ring. A schematic of the ring is shown in Fig. 2.

A laser beam was guided and focused by a series of mirrors and lenses and entered the ring through windows in the inner and outer vacuum chambers and in the thermal screen between them [1]. The laser beam and the ion beam were crossed at an angle of $90^{\circ}$. The laser power was monitored by means of a power meter placed behind the laser exit window. We used a Coherent 699-29 ring dye laser with rhodamine $6 \mathrm{G}$ as gain medium enabling the wavelength to be tuned in the region of the photodetachment thresholds in Fig. 1.

To measure the metastable level population as a function of time, a shutter was placed in the beam path of the laser. The interaction time of the laser light with the ions could be controlled by varying the opening time of the shutter. When photodetachment occurs, the resulting neutral sulfur atom is no longer confined by the electrostatic fields and continues along a straight line (see Fig. 2) towards a triple stack microchannel plate detector (MCP). The population was then probed by opening the shutter for fixed time

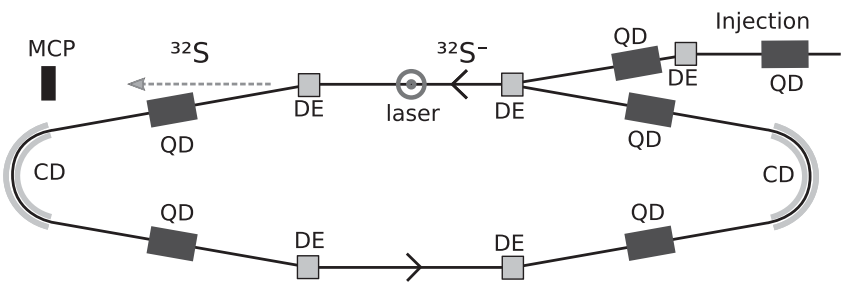

FIG. 2. A schematic of the main ion optical elements of the electrostatic ion storage ring viewed from above where CDs are $160^{\circ}$ cylindrical deflectors, DEs are $10^{\circ}$ deflectors, and QDs are quadrupole doublets. The circle indicates where the laser light crosses the ion beam. Neutral atoms formed by laser detachment of $10 \mathrm{keV}^{32} \mathrm{~S}^{-}$ions leave the straight section along the gray arrow and are counted with high efficiency by the MCP detector. 


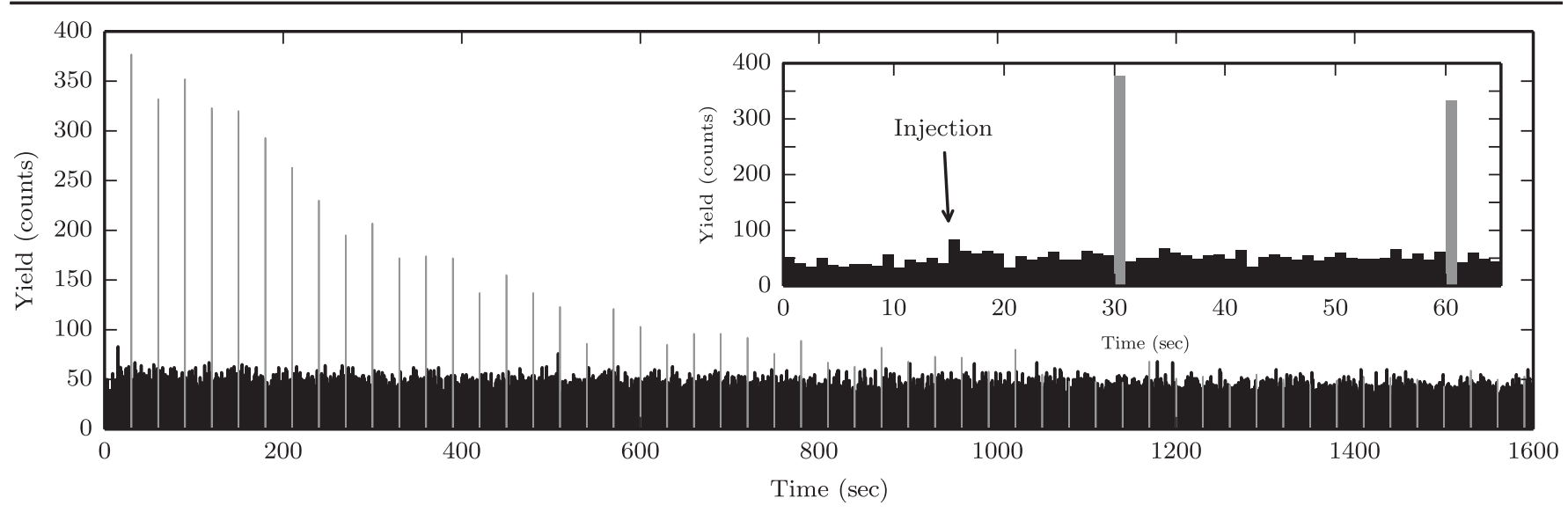

FIG. 3. The number of neutrals recorded on the MCP as a function of time after injection. The data were recorded for seven ion-beam injections. The inset shows the first $65 \mathrm{sec}$. For illustrative purposes, events recorded when the laser shutter is open are shown in gray. The ions are injected at $t=15 \mathrm{sec}$, as indicated by the arrow in the figure.

durations at regularly spaced time delays after injection while continuously recording the yield of neutrals impinging on the MCP detector. Figure 3 shows the data from probing the $3 p^{52} P_{1 / 2}^{o}$ population in ${ }^{32} \mathrm{~S}^{-}$as a function of time. These data were accumulated with seven consecutive ion-beam-pulse injections. Here, a photon energy of $2.03 \mathrm{eV}$, which is sufficient for photodetachment of ions in the metastable but not in the ground-state level, was chosen. The time of ion injection in the ring is indicated in the inset in Fig. 3. Before injection, the detector count rate is typically $6-8 \mathrm{~Hz}$. The excellent vacuum conditions are manifested by the fact that the count rate after injection of a $10 \mathrm{keV}, 10 \mathrm{nA},{ }^{32} \mathrm{~S}^{-}$beam only increases by a small amount (see Fig. 3 inset).

The depletion of the metastable level population by the laser was first investigated by changing the length of the laser pulses while keeping the power and number of pulses constant. In a second step, the laser power and pulse length were decreased until the laser-induced photodetachment loss rate became negligible. The maximum electric field strength to which the ions are exposed in the ring is about $1 \mathrm{kV} / \mathrm{cm}$. Thus, the effects of field detachment through tunneling [27] and decay induced by Stark mixing are completely negligible [28].

The measured effective decay rate $\Gamma_{\text {tot } 1 / 2}$ is a sum of the spontaneous radiative decay rate $\Gamma_{\mathrm{sp}}$, the pressuredependent rate at which the ions are lost due to collisions with residual gas $\Gamma_{\text {coll }}(p)$ and other (unknown) machineinduced loss mechanisms $\Gamma_{\text {other }}$,

$$
\Gamma_{\text {tot }, 1 / 2}=\Gamma_{\mathrm{sp}}+\Gamma_{\text {coll }, 1 / 2}(p)+\Gamma_{\text {other }} .
$$

In order to study the influence of the collisional rate $\Gamma_{\text {coll, } 1 / 2}(p)$, a series of measurements at different pressures were made. The results at two different pressures can be seen in Fig. 4. Increases in pressure were achieved by heating the cryogenic vacuum chamber slightly. Since the vacuum is out of reach of standard vacuum gauges, the decay rate of the stored ion beam is used as a relative measurement of the pressure. By tuning the laser wavelength below $596.9 \mathrm{~nm}$, photodetachment from both ${ }^{2} P^{o}$ fine structure levels occurs, and the recorded signal follows a double exponential decay with a fast and a slow component corresponding to the effective lifetimes of the metastable and ground-state ions, respectively. The residual gas was measured to be completely dominated by $\mathrm{H}_{2}$ at base pressure (at $13 \mathrm{~K}$ ) and also at the slightly raised pressures obtained when heating the ring to 15 or $17 \mathrm{~K}$.

In the analysis, we assume that the decay rate of the short-lived component when probing both levels (at $\lambda=592 \mathrm{~nm}$ ) and when probing only the excited metastable level (at $\lambda=610 \mathrm{~nm}$ ) is the same. Therefore, we fitted the data by minimizing $\chi^{2}$ for all data points belonging to both data sets together. The fitting functions are given in Fig. 4. The fitted fast versus slow components are displayed in the Stern-Vollmer plot of Fig. 5. We obtain the spontaneous radiative lifetime $1 / \Gamma_{\mathrm{sp}}=503 \pm 54 \mathrm{sec}$ by taking the inverse of the intercept of the straight line with the vertical axis [29]. In principle, we cannot know if the beam losses at base pressure are due to residual-gas collisions, machine losses, or a combination of both. However, at base pressure, we find a storage lifetime for ground-state ${ }^{32} \mathrm{~S}^{-}$ions of $939 \pm 27 \mathrm{sec}$, and in the top panel of Fig. 4, we see that there is still a stored beam after more than one hour. The common (unknown) loss rate $\Gamma_{\text {other }}$ cannot be larger than the measured beam-loss rate at base pressure. The maximum systematic error can, thus, be calculated by assuming $\Gamma_{\text {other }}=1.06 \times 10^{-3} \mathrm{sec}^{-1}$. This would only affect the lifetime value at the level of $1 \%$, which is negligible in relation to the uncertainty in our final result $\tau=503 \pm 54$ sec.

To benchmark our method at shorter time scales, we also measured the lifetimes of the corresponding spontaneous radiative decay in $\mathrm{Te}^{-}$and $\mathrm{Se}^{-}$. These belong to the same 


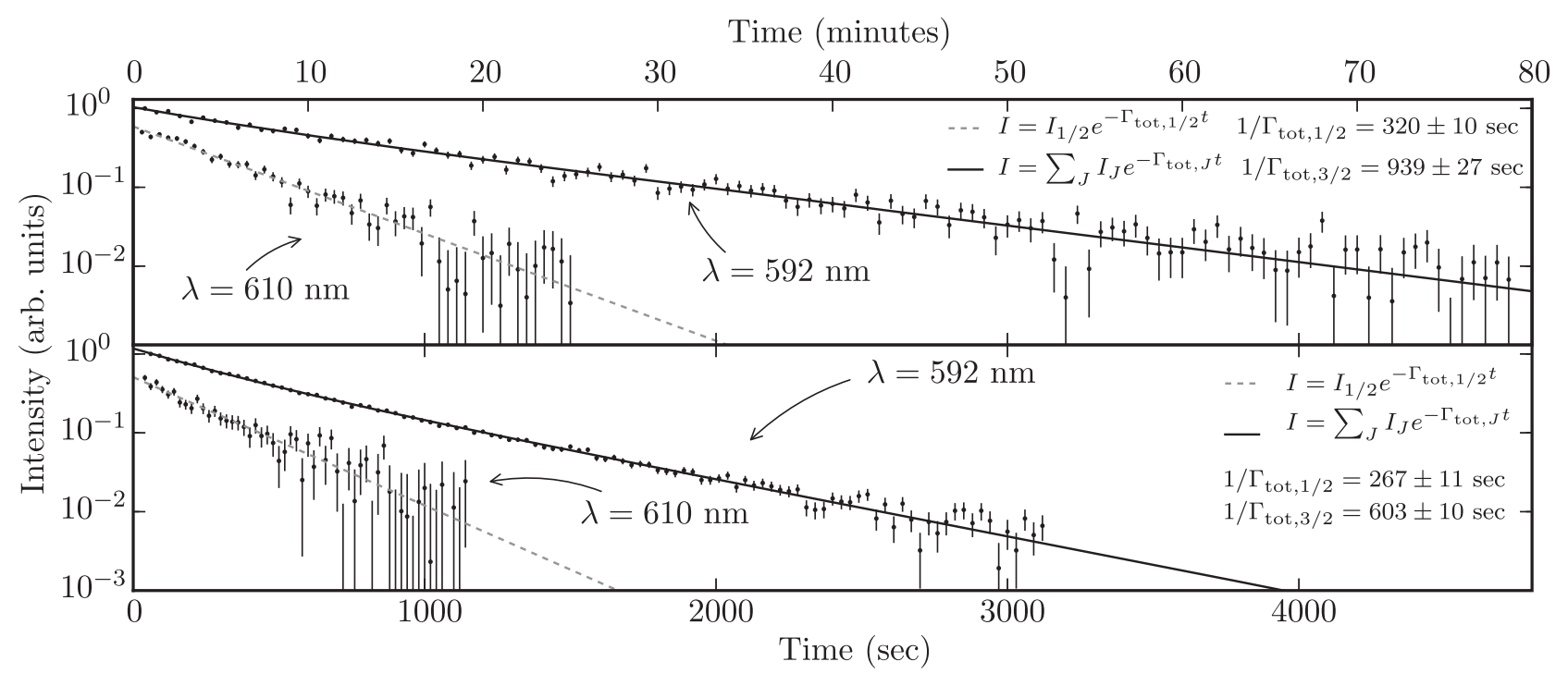

FIG. 4. Laser-probing measurements of metastable and ground-state-level populations as functions of time at base pressure at $13 \mathrm{~K}$ (top) and slightly elevated pressure at $15 \mathrm{~K}$ (bottom). The data points for $\lambda=610 \mathrm{~nm}$ exhibit a single exponential decay of the $J=1 / 2$ level population. When $\lambda=592 \mathrm{~nm}$, photodetachment from both levels $(J=1 / 2,3 / 2)$ occur, and the data follow a sum of two exponential functions (cf. text).

atomic group in the periodic table as $\mathrm{S}^{-}$. The present error bars for $\mathrm{Te}^{-}$and $\mathrm{Se}^{-}$are about a factor of 5 smaller than those of the only available previous experiments [8]. Both sets of experimental results are close to the theoretical results [8] and in agreement with each other, as shown in Table I. The theoretical calculations used the multiconfigurational Dirac-Fock method [30], included correlation effects, effects of finite nuclear sizes, as well as QED and Breit corrections [8]. The comparisons between the present experimental result for $\mathrm{S}^{-}, \mathrm{Te}^{-}$, and $\mathrm{Se}^{-}$and the predictions in Ref. [8] are favorable and indicate that this level of theory

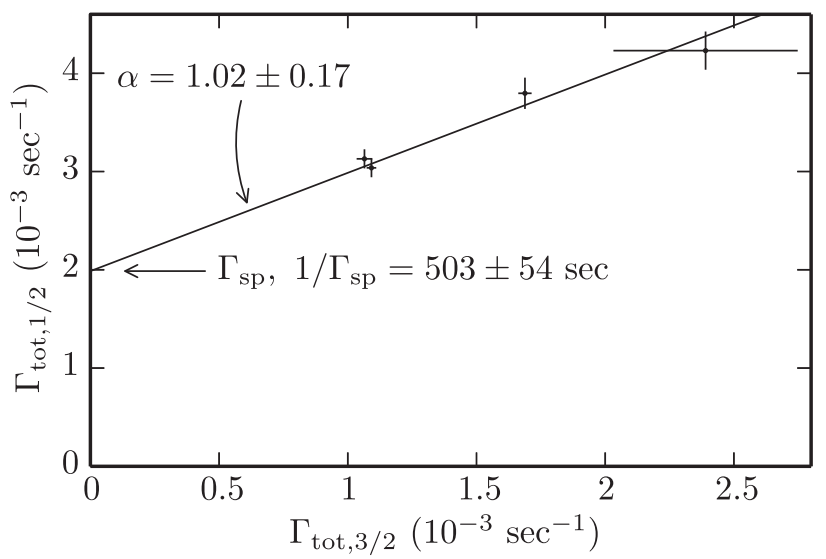

FIG. 5. Measured (effective) decay rate of the ${ }^{2} P_{1 / 2}^{o}$ level as a function of the measured effective decay rate of the ${ }^{2} P_{3 / 2}^{o}$ level (the ground state) in ${ }^{32} \mathrm{~S}^{-}$. The intrinsic spontaneous radiative decay rate is obtained by extrapolation to zero and is indicated by $\Gamma_{\mathrm{sp}}$. may be appropriate even for decay rates in the $10^{-3} \mathrm{sec}^{-1}$ range. For $\mathrm{Te}^{-}$and $\mathrm{Se}^{-}$, the measured metastable state decay rates are orders of magnitudes larger than the measured ion-beam loss rates which leads to higher precisions in these measurements compared to the one for $\mathrm{S}^{-}$. The $(1 / e)$ storage lifetime of the $10 \mathrm{keV} \mathrm{Te}^{-}$beam was, for example, measured to be $30 \mathrm{~min}$.

In conclusion, we have demonstrated that a newly constructed cryogenic ion storage ring can be used to store negative ion beams in the hour time domain. This allowed us to determine the lifetime of the $3 p^{52} P_{1 / 2}^{o}$ level in ${ }^{32} \mathrm{~S}^{-}$ to be $\tau_{\mathrm{sp}}=503 \pm 54 \mathrm{sec}$, which corresponds to $8.38 \pm$ $0.90 \mathrm{~min}$. This is, to our knowledge, the longest lifetime ever measured for a negatively charged atomic, molecular, or cluster ion. The technique is very general and can be applied to any kind of atomic anion with lifetimes from milliseconds to several minutes. In addition, it may easily be extended to studies of stabilities and cooling of molecular and cluster anions. As the systems are cooled

TABLE I. The experimental results for the lifetimes of the metastable $n p^{5}{ }^{2} P_{1 / 2}^{o}$ levels in $\mathrm{S}^{-}, \mathrm{Se}^{-}$, and $\mathrm{Te}^{-}$. Previous experimental and theoretical results are also given when available.

\begin{tabular}{lccc}
\hline \hline & \multicolumn{3}{c}{$\tau(\mathrm{sec})$} \\
\cline { 2 - 4 } Ion & Theory [8] & Experiment [8] & This work \\
\hline $\mathrm{S}^{-}$ & 437 & $(\cdots)$ & $503(54)$ \\
$\mathrm{Se}^{-}$ & 4.92 & $4.7(7)$ & $4.78(18)$ \\
$\mathrm{Te}^{-}$ & 0.454 & $0.42(5)$ & $0.463(8)$ \\
\hline \hline
\end{tabular}


in the ring, we may combine the advantages of storing ions at high velocities with the advantage of an environment which has a strongly reduced black-body radiation background. The latter gives unique access to studies of very weakly bound systems, and our new instrument $[1,2]$ also opens up many possibilities for action spectroscopy on cold molecules and for merged-beams collision studies. In some specific cases, it will be possible to probe the decay of individual quantum states by means of photodetachment via resonant states. A particularly interesting case here is the laser-cooling candidate $\mathrm{La}^{-}$, where the lifetimes of the ${ }^{3} F$ fine structure levels have been predicted to be in the range of hundreds of seconds [12].

This work was supported by the Swedish Research Council (Contracts No. 821-2013-1642, No. 621-20123662, No. 621-2011-4047, and No. 621-2013-4084) and by the Knut and Alice Wallenberg Foundation. We acknowledge support from the COST action CM1204 XUV/X-ray light and fast ions for ultrafast chemistry (XLIC). We thank Christophe Blondel for interesting discussions of the results.

*erba@fysik.su.se †schmidt@fysik.su.se

[1] R. D. Thomas et al., Rev. Sci. Instrum. 82, 065112 (2011).

[2] H. T. Schmidt et al., Rev. Sci. Instrum. 84, 055115 (2013).

[3] D. J. Pegg, Rep. Prog. Phys. 67, 857 (2004).

[4] T. Andersen, H. K. Haugen, and H. Hotop, J. Phys. Chem. Ref. Data 28, 1511 (1999).

[5] T. Andersen, Phys. Rep. 394, 157 (2004).

[6] C. Blondel, W. Chaibi, C. Delsart, C. Drag, F. Goldfarb, and S. Krger, Eur. Phys. J. D 33, 335 (2005).

[7] C. Blondel, W. Chaibi, C. Delsart, and C. Drag, J. Phys. B 39, 1409 (2006).

[8] P. Andersson, K. Fritioff, J. Sandström, G. Collins, D. Hanstorp, A. Ellmann, P. Schef, P. Lundin, S. Mannervik, P. Royen, K. C. Froese Fischer, F. Österdahl, D. Rostohar, D. J. Pegg, N. D. Gibson, H. Danared, and A. Källberg, Phys. Rev. A 73, 032705 (2006).

[9] R. C. Bilodeau and H. K. Haugen, Phys. Rev. Lett. 85, 534 (2000).

[10] C. W. Walter, N. D. Gibson, Y.-G. Li, D. J. Matyas, R. M. Alton, S. E. Lou, R. L. Field, D. Hanstorp, L. Pan, and D. R. Beck, Phys. Rev. A 84, 032514 (2011).

[11] C. W. Walter, N. D. Gibson, D. J. Matyas, C. Crocker, K. A. Dungan, B. R. Matola, and J. Rohlén, Phys. Rev. Lett. 113, 063001 (2014).

[12] S. M. O'Malley and D. R. Beck, Phys. Rev. A 81, 032503 (2010).
[13] M. Yasuda and H. Katori, Phys. Rev. Lett. 92, 153004 (2004).

[14] B. B. Jensen, H. Ming, P. G. Westergaard, K. Gunnarsson, M. H. Madsen, A. Brusch, J. Hald, and J. W. Thomsen, Phys. Rev. Lett. 107, 113001 (2011).

[15] S. S. Hodgman, R. G. Dall, L. J. Byron, K. G. H. Baldwin, S. J. Buckman, and A. G. Truscott, Phys. Rev. Lett. 103, 053002 (2009).

[16] D. Zajfman, O. Heber, L. Vejby-Christensen, I. Ben-Itzhak, M. Rappaport, R. Fishman, and M. Dahan, Phys. Rev. A 55, R1577 (1997).

[17] H. T. Schmidt, H. Cederquist, J. Jensen, and A. Fardi, Nucl. Instrum. Methods Phys. Res., Sect. B 173, 523 (2001).

[18] S. P. Møller, Nucl. Instrum. Methods Phys. Res., Sect. A 394, 281 (1997).

[19] R. von Hahn et al., Nucl. Instrum. Methods Phys. Res., Sect. B 269, 2871 (2011); in Proceedings of the 10th European Conference on Accelerators in Applied Research and Technology (ECAART10).

[20] J. Bernard, G. Montagne, R. Bredy, B. Terpend-Ordaciere, A. Bourgey, M. Kerleroux, L. Chen, H. T. Schmidt, H. Cederquist, and S. Martin, Rev. Sci. Instrum. 79, 075109 (2008).

[21] T. Andersen, L. H. Andersen, P. Balling, H. K. Haugen, P. Hvelplund, W. W. Smith, and K. Taulbjerg, Phys. Rev. A 47, 890 (1993).

[22] U. V. Pedersen, M. Hyde, S. P. Møller, and T. Andersen, Phys. Rev. A 64, 012503 (2001).

[23] A. Wolf, K. G. Bhushan, I. Ben-Itzhak, N. Altstein, D. Zajfman, O. Heber, and M. L. Rappaport, Phys. Rev. A 59, 267 (1999).

[24] P. Reinhed, A. Orbán, J. Werner, S. Rosén, R. D. Thomas, I. Kashperka, H. A. B. Johansson, D. Misra, L. Brännholm, M. Björkhage, H. Cederquist, and H. T. Schmidt, Phys. Rev. Lett. 103, 213002 (2009).

[25] A. Ellmann, P. Schef, P. Lundin, P. Royen, S. Mannervik, K. Fritioff, P. Andersson, D. Hanstorp, C. F. Fischer, F. Österdahl, D. J. Pegg, N. D. Gibson, H. Danared, and A. Källberg, Phys. Rev. Lett. 92, 253002 (2004).

[26] SNICS-Source of Negative Ions by Cesium Sputtering, National Electrostatic Corporation, USA.

[27] A. Litherland and M.-J. Nadeau, Nucl. Instrum. Methods Phys. Res., Sect. B 99, 546 (1995); application of Accelerators in Research and Industry ' 94.

[28] A. Khadjavi, A. Lurio, and W. Happer, Phys. Rev. 167, 128 (1968).

[29] S. Mannervik, A. Ellmann, P. Lundin, L.-O. Norlin, D. Rostohar, P. Royen, and P. Schef, Phys. Scr. T119, 49 (2005).

[30] F. Parpia, C. Fischer, and I. Grant, Comput. Phys. Commun. 94, 249 (1996). 